Proceedings of the 2011 Winter Simulation Conference

S. Jain, R.R. Creasey, J. Himmelspach, K.P. White, and M. Fu, eds.

\title{
REPRESENTATION OF HUMANITARIAN AID / DISASTER RELIEF MISSIONS WITH AN AGENT BASED MODEL TO ANALYZE OPTIMAL RESOURCE PLACEMENT
}

\author{
Andrew Turner \\ Dr. Santiago Balestrini-Robinson \\ Dr. Dimitri Mavris \\ Georgia Institute of Technology \\ 281 Ferst Drive \\ Atlanta, GA 30332, USA
}

\begin{abstract}
An Agent Based Model was developed to help analyze the importance of the size, number, operating time, and placement of resources dispensaries and processing centers in Phase II of a Humanitarian Aid / Disaster Relief (HA/DR) mission. The ABM developed takes into account population density, social economic attributes of the population, ethnicity makeup of the population, crime rates, resource needs, medical needs, and migration of the population after a disaster. The model was developed in NetLogo 4.1 to be a flexible analysis tool that can represent a variety of generic populated areas in need of humanitarian assistance. The model was analyzed varying 11 factors and tracking 6 responses using a 128 case Nested Latin Hypercube Design and a 60 case Robust Screening Design with 20 repetitions. The analysis determined the number of distribution and processing centers to be the major driving factor in the responses studied.
\end{abstract}

\section{INTRODUCTION}

Humanitarian assistance and disaster relief (HA/DR) missions continue to be a major part of military missions around the globe as evidenced by the humanitarian disasters in Africa, Haiti, Pakistan, Turkey, and Japan. Despite this reality, very few models have been developed that capture the broad complexities of the HA/DR mission. The primary focus of many models is the analysis and optimization of the logistic networks and the pre-positioning of resources. These models often stop short of modeling the flow of resources down to the individual. Though these studies are critical for a successful HA/DR mission, the process of delivering resources from the arriving shipments to the people has shown to be very problematic as evidenced by the crises in Haiti and Africa. In the aftermath of the 7.0 Richter Scale magnitude earthquake that struck Haiti in 2010 there were a large number of accounts of looting, gun-fights, and riots due to the lack of security enforcement and resource shortages despite resources sitting just off the coast of Port-au-Prince (Daily Telegraph. 2010a; Daily Telegraph. 2010b). It is not enough to just deliver relief to the region it must also be distributed throughout the population in a safe, orderly and secure manner. The most common approach is to set up centers in order to provide the population with the required resources and services. The primary questions we ask are: How should these centers be set up to best meet the needs of the people? Should there be fewer centers with greater capacity or should there be more low capacity centers? How does the total daily operation time affect the distribution of resources and services? And finally, how do these incite different levels of insecurity and population migration? 


\section{HA/DR DECOMPOSITION}

The HA/DR mission is a vast, complex problem incorporating logistic networks, structural engineering, social sciences, and public health. No one model can capture every aspect of the problem. However, a set of models can be developed that analyze aspects of the problem to understand how to perform the mission better. The HA/DR problem must be explained and scoped before a model can be developed. The primary effects of a natural disaster is the destruction of infrastructure and the loss of life. These two primary effects generally lead to three major results, namely limited resources, spikes in need for medical treatment, and an overall reduction quality of life of the affected population. In order to return the region to its former status, resources and people must be brought in to distribute critical resources, maintain security, reestablish the infrastructure, and provide medical care. These resources and people are brought into the region from a larger logistic network where they then operate on in a smaller regional logistic network. A generic regional logistic network is show in Figure 1. As can be seen in the figure below, resources and people can only enter the area in three ways: sea port, air port, and roads. After the disaster the capacity of these routes could be severely diminished. From these nodes volunteers, aid workers and military personnel will deliver water, food, power, and medical care to the people. Note that additional resources and services may be required depending on the scenario. Having defined the entire regional network, the problem is then scoped down for modeling. The ABM developed will only focus on the delivering of water, food and medical relief to the population. This is indicated by the blue shading in the figure below. The main assumption behind this model is that necessary the resources are able to reach the distribution centers and processing centers, i.e., breaks in the logistics network are not modeled. The model does not include utilities, i.e., electric power restoration and supply is not modeled.

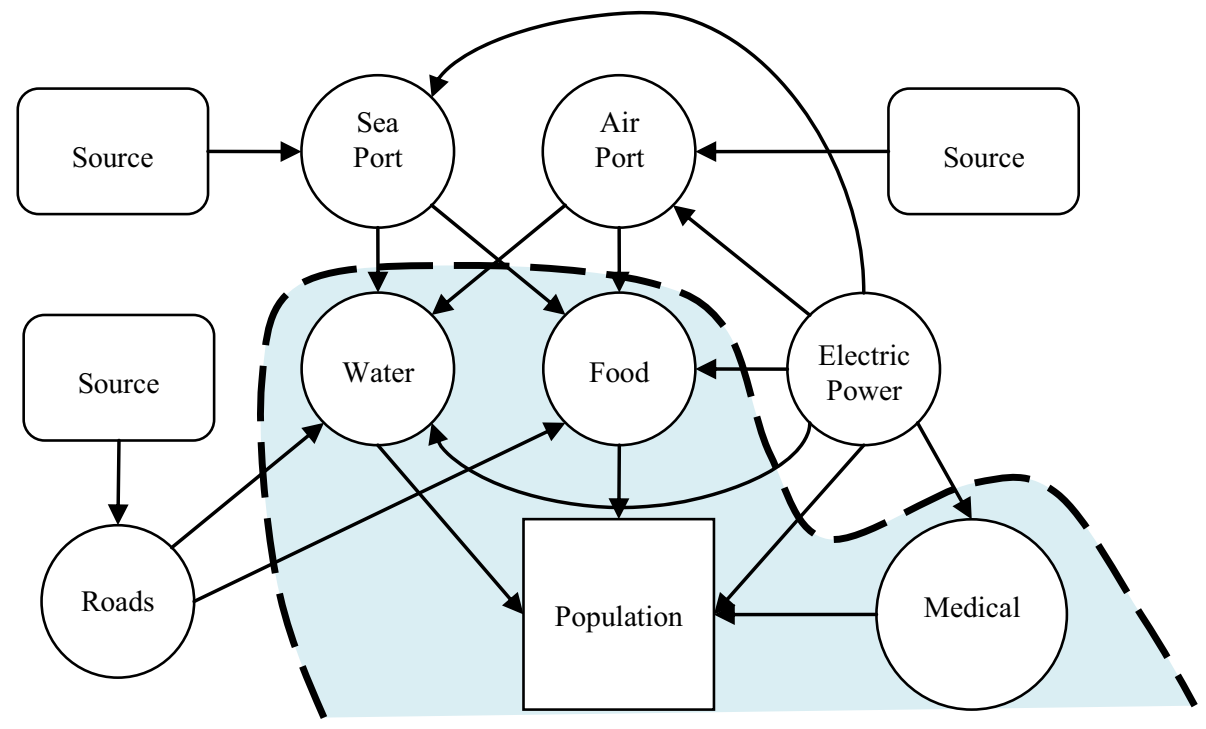

Figure 1: Regional Network

\section{HA/DR MODEL}

In order to develop the proper model of a HA/DR mission, one must consider the following aspects of the community in distress: population density, ethnic makeup and tensions, social attributes, resources availability and desire, crime rates, and the location and makeup of processing and dispensary centers. The model was developed in NetLogo 4.1, a freeware agent-based and system dynamics modeling framework developed by The Center for Connected Learning and Computer-Based Modeling at Northwestern University (Wilensky 1999). 


\section{Turner, Balestrini-Robinson and Mavris}

\subsection{Population Density}

Modeling every individual in the population as an agent would be infeasible from a computational standpoint, so a method for simplifying the pertinent characteristics of the population had to be devised. To confront this issue a combination of Cellular Automata and Agent Based Models was used. The environment that the model operated on was split up into a discrete set of patches, in this case $32 \times 32$. These patches represented a geographical area of a given physical dimension represented in meters. Each patch contains information on the number of individuals living in the area in addition to other information pertinent to the societal and geographical characteristics of the area, these are discussed in more detail in the following sections. The total number of people in the model is predefined and then is randomly distributed throughout the map. The model can be easily modified to have higher resolution-i.e., higher discretization - of the environment. The cellular automata portion of the model, i.e., the population, interacts with the environment thought the production of agents. An agent is produced by the patch in order to gather resources, travel to receive medical attention, to migrate, or to commit a crime. These actions will be discussed in the following sections. An agent represents a group of people from that patch, this group can range in size between one person up to the number of people residing in that patch.

\subsection{Ethnic Considerations}

Not all HA/DR scenarios have a major focus on ethnic conflict. On the other hand, some HA/DR missions are a direct result of, or can be affected by, ethnic tensions. For this reason the ethnic makeup and corresponding tensions of a region must be considered in order to study how conflict arises.

Let's assume a scenario that contains two ethnic groups (A and B). In addition assume these two groups have a history of violence. If resource center locations force mixing of the two groups, a resulting increase of crime will occur. For this reason the model includes the ethnic group breakdown for each patch. In addition the model includes an inter-relationship matrix that determines how well the different ethnic groups interact. This will have an important impact when studying the evolution of crime.

\subsection{Social Attributes}

Social attributes help to better define the makeup of the population. This data is stored in the patch. Some examples of these attributes are poverty rates, education rates, and employment. These metrics will play a major role in calculating crime. The social attribute data can be attained from census data or similar databases.

\subsection{Crime}

Crime is an important and complicated aspect of the model. Crime ran rampant in a wide variety of HA/DR missions, especially in Haiti after the 2010 earthquake. This was a result of insufficient security forces and lack of vital resources. Understanding how the placement of centers impacts the crime of the affected area is essential to produce a comprehensive and satisfactory plan for HA/DR missions.

The occurrence of crime is a result of a number of different factors (Malleson et al. 2009). These factors range from socioeconomic status and education to criminal punishment and the reinforcing nature of crime (Buonanno et al. 2008; Malleson et al. 2009; Pablo et al. 2002; Schrag et al. 1997; Scorzafave et al. 2009). In addition, crime rates and the sources of crime vary across different societies (Malleson et al. 2009). For a measure of impact on crime rates the reader is referred to (Buonanno et al. 2008; Malleson et al. 2009; Pablo et al. 2002; Schrag et al. 1997; Scorzafave et al. 2009).

Due to the wide range of factors contributing to crime and the varying impacts across different societies an expandable and adaptable crime model was developed. This model is composed of two phases. The first phase is the probability of a patch to create a crime agent. The second phase takes place when the crime agent travels in a random walk until it commits a crime. A crime agent will commit a crime based on a separate probability. This probability is based on the characteristics of that agent and the patch 


\section{Turner, Balestrini-Robinson and Mavris}

where that agent currently resides. The probability of a patch to create a crime agent is presented in equation (1). The probability of a crime agent to commit a crime is presented in equation (2).

The equation displayed in (1) is composed of six elements. The first is the dominating coefficient multiplying the whole equation, DN. This coefficient allows the probably to distinguish between day and night. The second part is a summation of the impact of social attributes on crime with their corresponding weightings. The third part is a measure of the desire level of the patch. If the people are without critical resources they are more likely to engage in criminal acts out of desperation. The fourth is a measure of an ethnic group's tendency towards crime. The hypothesis is that one ethnic group may be quicker to resort to crime than another. This ethnic consideration may not always be present and can be zeroed if deemed irrelevant. The fifth part is the factor that describes the reinforcing nature of crime (Buonanno et al. 2008; Schrag et al. 1997). The sixth, and final part is a measure of the impact on the presence of security forces. The equation displayed in (2) is the probability a crime agent will commit a crime, and it is similar in form to (1). The first significant change is the second summation term. This is a measure of the crime agents' desire for resources and the potential victims' desire for resources. The potential victim is the current patch that the crime agent is on. The next difference is in the next term. Here the ethnicity of the crime agent and the potential victim plays a role. Depending on the ethnic relationship this could increase or decrease the probability of a crime being committed. The final difference is that there is no self reinforcing nature of crime once the crime agent is produced. Due to limitations in space, not all the coefficients can described, but in general, the equation can be described a weighted sum, where a coefficient weighs the impact that each term in the sum has on the probability of occurrence. Great care must be taken to ensure the resulting probability is realistic, i.e., it ranges between 0 and 1 , and it correctly represents reality.

$$
\begin{aligned}
& P=D N\left[\sum_{i=1}^{n} a_{i} \frac{\left(100-S A_{i}\right)}{100}+\sum_{j=1}^{m} a_{n+j} \max \left[0,1-\frac{R L_{j}}{R D_{j}}\right]+\sum_{k=1}^{o} a_{n+m+k} E t h_{k}+c * C C-\sum_{l=1}^{e} E \frac{1}{\max \left[1, R_{l}^{2}\right]}\right] \\
& P=D N\left[\sum_{i=1}^{n} a_{i} \frac{\left(100-S A_{i}\right)}{100}+\sum_{j=1}^{m} a_{n+j}\left(1-\min \left[1, \frac{R L_{j}}{R D_{j}}\right]\right) \frac{R L_{v}}{R D_{v}}+E_{E t, E t} E t h_{v}-\sum_{l=1}^{e} E \frac{1}{\max \left[1, R_{l}^{2}\right]}\right]
\end{aligned}
$$

\subsection{Resource and Medical Needs}

Every patch will contain information on the amount of resources that exist on that patch. Examples of this could be liters of water and calories of food. The patch will consume the resources at a specified rate based on the size of its population. This will continue to change until the patch reaches the desire threshold. Every patch has a different desire for resources, assigned randomly from an initial distribution. If the patch reaches the desire threshold then the patch will create a gathering agent. This gathering agent will consist of a percentage of the people on the patch. The percentage is a defined input to the model. This gathering agent will then travel to the nearest resource dispensary to acquire the desired resources and return them to its home patch. Every patch will also have needs for services such as medical treatment. Medical needs are defined as a uniform percentage of the population across all the patches. Each patch has an associated time at which point the medical need will occur. Once sufficient time has elapsed an agent is produced by the patch and travels to receive medical treatment.

\subsection{Dispensing and Processing Centers}

An important distinction needs to be made between a processing center and a dispensing center. A processing center is a center that the people visit to have some service done for them or to them. There is nothing physical that the people leave the center with. The people must report here to receive what is 


\section{Turner, Balestrini-Robinson and Mavris}

needed. Examples of this include hospitals or vaccination clinics. In contrast, a dispensing center is one that gives the people a material good that can be carried back to their home. The people could either report to the center personally to receive the good or have someone retrieve the good for them. Examples of this are food and water. Every center has a maximum throughput capacity and a limited supply. The throughput capacity is determined by the number of people that can be processed at once and the time it takes to process an individual. The throughput capacity of the resource and processing centers will differ greatly. For example, more time is required to treat medical needs than to hand out food. In addition, medical experts are in shorter supply than people who can hand out food. The agent will arrive at the center and get into a line. They will wait until they are processed, the center runs out of resources, or the center closes. Once this occurs the agent returns home. If their needs are not meet, they will return at the start of the following day.

\subsection{Migration}

The final element to be modeled is migration. The premise of this portion of the model is that if people are suffering - e.g., due to crime, lack of resources or services, ethnic tensions - and there are areas with better conditions, or a promise for better conditions, they are likely to leave and move to the more promising area. HA/DR missions do not consider migration a positive outcome of their mission, and desire to minimize the impact on the population, including their tendency to migrate and relocate.

The equation shown in (3) describes the elements that compose the calculation that determines the goodness of a given patch, $P_{\text {good. }}$ The $a, b, c$ and $d$ terms are multipliers for each of the terms. The term $r_{p}$ is a relaxation constant. If the term is zero the goodness of the patches does not change from one time step to the next. If it is set to one, it will have no memory of the goodness from the prior step. The first term is the summation over the $m$ Social Attributes of patch, e.g., education, employment, etc. The parameters $C T, C G M$ and $C M$, are the counters for the number of crimes committed by crime agents from other patches on the patch, how many successful crimes crime agents from that patch have conducted in other patches, and the number of gatherers that have been mugged on that patch respectively. The parameter $t$ is time. Pop and PopAreaRatio are the number of people residing on that patch and the ratio between total area and total population respectively. This term helps quantify overcrowding in relative terms. The final term is a measure of how many resources that patch is lacking, where $\left(R_{D}\right)_{i}$ is the $i^{\text {th }}$ resource desired and $\left(R_{P}\right)_{i}$ is the amount of that resource present. The coefficient $e_{i}$ is a measure of the weighing given to that resource by the population on that patch.

$$
P_{\text {good }}=\left(1-r_{p}\right) P_{\text {good }}+r_{p}\left[\begin{array}{c}
\frac{a}{m} \sum_{i=1}^{m} S A_{i}-b \frac{(C T-4 C G M-C M)}{t} \ldots \\
-c(\text { Pop } \times \text { PopAreaRatio })-d \sum e_{i} \frac{\left(R_{D}\right)_{i}-\left(R_{p}\right)_{i}}{\left(R_{D}\right)_{i}}
\end{array}\right]
$$

The goodness of all the patches is calculated at every time step, and it is compared to a parameter that represents the value of the property in that patch. This parameter accounts for the fact that if people have a highly valuable property, they are more likely to remain there rather than if they have no material reason to stay.

Once a group of people in a patch decides to migrate, they look for a patch that is at least a certain amount better than their patch and has members of their ethnicity. Then a migration agent is created and that agent traverses to the new location. The size of this agent can range in size from one to the number of people of a given ethnicity residing on the given patch. 


\section{ANALYSIS}

Once the model was created, a Design of Experiments (DOE) could be executed to determine how the centers should be set up to best meet the need of the people affected by the disaster. The first step of this process is to define the control variables of the model and the subsequent ranges.

\subsection{Design Ranges}

Design ranges for the center control variables can be developed from the operations used by the US Army Corps of Engineers (USACE). USACE uses three different types of resource centers. They operate by loading resources into cars that pass through 4, 2, and 1 lanes of cars, respectively. This case is amenable to a domestic HA/DR operation, e.g., Hurricane Katrina. They make the assumption that each lane can process 5,000 people every 12 hours. Each car is assumed to represent 3 people and is given 1 day of supplies for each person. The centers operate for a total of 12 hours a day (State of Missouri - State Emergency Management Agency). With this information in addition to basic approximations, design ranges for the 10 input variables were defined and are shown in Table 1. In addition to the variable ranges, the placement of security forces have two different defined architecture scenarios. One places additional security at the centers and the other places security pseudo-randomly throughout the region using a layout function.

Table 1: DOE Variable Ranges

\begin{tabular}{l|c|cc}
\hline \multicolumn{1}{c|}{ Variable } & Short Name & Lower & Upper Bound \\
& & Bound & \\
\hline Population & Pop & 50,000 & 200,000 \\
Center Operation Time & Op Time & $8 \mathrm{Hr}$ & $18 \mathrm{Hr}$ \\
Resource Limit per Dispensary & DRPP & $2,000 \mathrm{ppl}$ & $20,000 \mathrm{ppl}$ \\
Dispensary Lines & Dist Lines & 1 & 6 \\
Time to Dispense per Person & DTPP & $20 \mathrm{sec}$ & $30 \mathrm{sec}$ \\
Number Resource Centers & \# Dist Ctrs & 1 & 20 \\
Supplies Dispensed & Amnt Distributed & 1 Day & 10 Days \\
Number Process Centers & \# Proc Ctrs & 1 & 8 \\
Processing Lines & Proc Lines & 2 & 10 \\
Time to Process one Person & PTPP & 5 minutes & 60 minutes \\
\hline
\end{tabular}

\subsection{Design of Experiments}

The DOE selected for the analysis of the model utilized a 128 case Nested Latin Hypercube Design (NLHD) developed by Prof. Peter Qian (Qian 2009) plus three different variations of a 20 case Robust Screening Design (RSD) (Jones and Nachtsheim 2010). The 20 RSD cases were scaled to span 50\%, 75\% and $100 \%$ of the design space, for a total of 60 cases, so as to assess the predictive capability of the models generated by the NLHD points. Each of the 188 cases were executed 20 times with a different random seed to obtain statistically significant results.

The 128 NLHD cases allow the designer to run increasingly larger orthogonal blocks, i.e., the first block has 16 cases which are orthogonal, and when the additional blocks of 16, 32, and 64 cases are included, the correlation of the aggregated design remains at a minimum value. This allowed the team to obtain early results for analysis, as opposed to waiting for the whole experiment to be finished. This analysis helped the team ensure the model was operating correctly before all resources have been committed. Including the RSD and NLHD cases, the 20 repetitions, a total of 3,760 cases were executed. In addition, further executions of the model were conducted to analyze two different security enforcement scenarios.

The RSD cases were used to validate the goodness of the regressions based on the NLHD cases. The sequentially increasing span of the RSD allows for the testing of how well the model interpolates and extrapolates to the corners of the design space. The authors extend their thanks to Tom Donnelly from JMP-SAS for providing the knowledge and the designs. 


\subsection{Results}

The data collected from the model included resource needs, processing needs, crime rates, and migration at 6 points over three days (every 12 hours of simulation). The data was analyzed using JMP, a data visualization and statistical analysis software developed by SAS. The analysis shed light onto the question of how the centers should be set up, both in terms of quantity and characteristics. It was found that the number of centers was a more critical factor than their number of lines, i.e., it is better to have more smaller centers than fewer bigger centers. This result does not include the ease of supplying the centers, which would favor fewer larger centers.

Figure 2 depicts a prediction profiler as produced by JMP. The abscissa lists the controllable factors (i.e., inputs) and the ordinate the responses (i.e., outputs). This plot depicts how the variation of each of the inputs impacts each of the outputs, assuming all other inputs are fixed. The triangles are an indication of the rate of change of the response for that given input. Due to spatial constraints, the only responses depicted are water and medical need of the population, average and maximum wait times for the population to obtain resources and services, crime and migration after $1 \frac{1}{2}$ and 3 days after the mission began. Relations with a steeper curve indicates that the factor has a more significant impact on that response. For example, Maximum Wait Time for a Service after 3 days, i.e., MaxWaitProc (3d), is greatly impacted by the time the centers are opened (Time Open). Since the processing centers (medical and vaccination) take much longer to process a person when compared to the distribution centers, longer operation times result in longer queues. This in turn leads to longer wait times before a patient receives treatment. At the same time, it seems that there is no benefit in keeping the centers open for more than $12 \mathrm{hrs}$, since the need for medical service, i.e., Medical (1.5d) and Medical (3d), does not decrease much more after that point.

It was determined based on sensitivity analysis, that during HA/DR missions it is better to set up several small centers with low capacity throughout a region as opposed to few large centers with high capacity. This result is in agreement to what is to be expected, since the economies of scale of having fewer larger centers and their increased logistic efficiency cannot be currently calculated by the model. Nonetheless, this result is of importance. If the assumptions in terms of population distribution and resource need are correct, fewer super-centers are worse at satisfying the need than fewer smaller centers, even if the total capacity of the small centers is not as great as that of the super-center. This has intrinsic assumptions that the population is constrained in its mobility and the logistic support of the centers is not a driving element of the decision.

An original result from this work is the effect of the distribution and resource centers have on the crime in the area and the migration of the population. The most significant impact on crime is the number of distribution centers. The analysis shows that more centers results in fewer crimes are committed. In one aspect this is intuitive. People will travel less when more centers are available, therefore it is less likely that they will have their HA resources stolen from them. With only two distribution centers, crime rate after 3 days is estimated to be approximately 400 incidents for a population 185,000 (on the higher end of the range studied), whereas with 10 centers that decreases to 140 incidents after 3 days, and with 15 centers, less than 75 crime incidents. Amount distributed and number of distribution lines had an impact on reducing crime, indicating that providing the population with more resources reduces their incentive to commit crimes. 
Turner, Balestrini-Robinson and Mavris

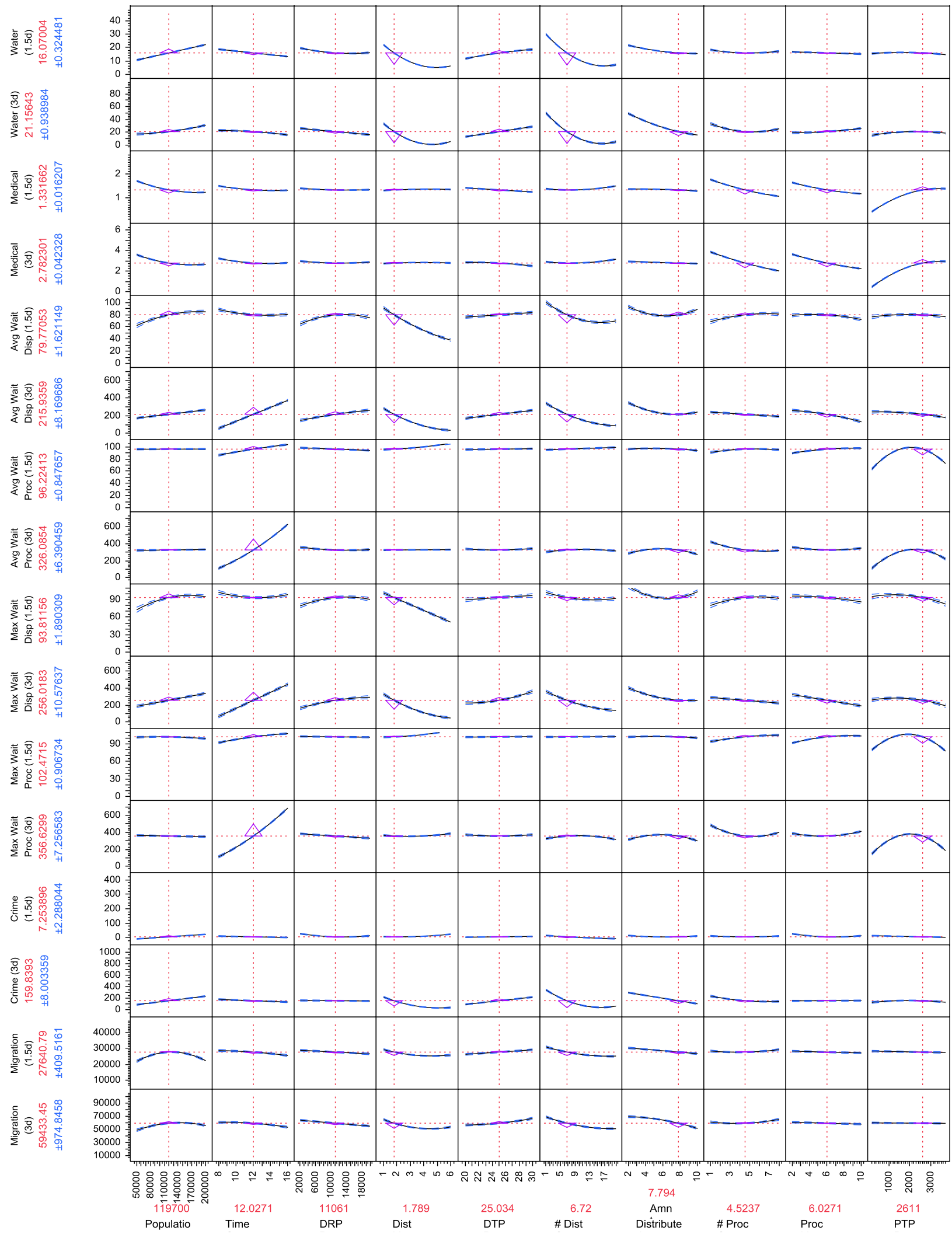

Figure 2: Prediction Profiler based on Polynomial Regressions 


\section{Turner, Balestrini-Robinson and Mavris}

Population migration was affected by the same factors that affected crime, but the amount distributed had a larger impact on reducing the migration of people. The reader is reminded that population migration is measured as the number of people that migrated times the distance they migrated over the time span of interest. Therefore, the more people, the more migration, but people have an incentive to not move to areas that are already overpopulated, so very high populations (effectively population density since the area remained the same) tend to deter migration. As with crime, the number of processing centers had effectively little to no effect. Unlike with crime though, there seems to be a monotonic impact to the number of centers, as in more centers, less migration. Amount distributed seemed to have an accelerating impact, as in the more distributed, the more the impact on reducing migration.

The crime and migration results are undoubtedly impacted by the mathematical models and distributions used to characterize them. The reader is reminded that the purpose of an agent-based model, as used in this application, is to study the emergent behavior that arises from the interaction of the simple agents. In this case, the results proved to be fairly linear and agreeable with intuition, nonetheless, this is something that could not have been predicted before the analysis was done.

The impact of enforcement location was analyzed by development of a separate scenario. The original scenario placed 16 enforcement agents pseudo-randomly around the population, dispensary and processing centers using a layout function based on a magnetic-spring model. The follow up scenario placed the enforcement agents at the centers. Preliminary analysis showed that this had little effect on the model. This result was to be expected since the model assumes that the centers operate in a civil manner, i.e., no crime occurs within the centers. Therefore, the centers gain nothing from the additional security. In addition, the regions surrounding the centers have their needs fulfilled better than outlying regions. In both scenarios the center region fairs well due to a concentration of centers and enforcers. Selected outlying regions suffer more from crime and migration. Further analysis will be required to determine if placing additional enforcers in the more distant areas will benefit the region as a whole.

Another useful approach from having an analysis capability is that the mission space can be evaluated as a function of the desired performance. Figure 3 depicts two contour profilers, in essence a constraint diagram over the inputs. The constraints are based on required minimum and/or maximum thresholds for the responses. In the figure above we have the available option space for the time the centers should be open as a function of the size of the population. The white region is the area where the requirements will be satisfied, in this case maximum wait times, crime rates and population migration. In this case, the constraint based on the average wait time to be processed after 3 days is not affected by the time the centers are opened, whereas the average time waiting to receive resources after 1.5 days does. If one is to examine that last constraint (highlighted in purple on the bottom right corner), it is clear that as the population grows, the centers should be open for longer. The processing centers exhibit the opposite behavior (which in turn has to do with the nature of the metric) in that if people are not to wait on average more than $60 \mathrm{~min}$ to receive treatment, the centers should not be opened for more than $12 \mathrm{hrs}$, since then the people waiting in line would leave and come back the next day. This is clearly a product of the way the metric is computed, but this highlights the very real issue that when measuring humanitarian missions, how something is defined and calculated may not produce the intended results. 

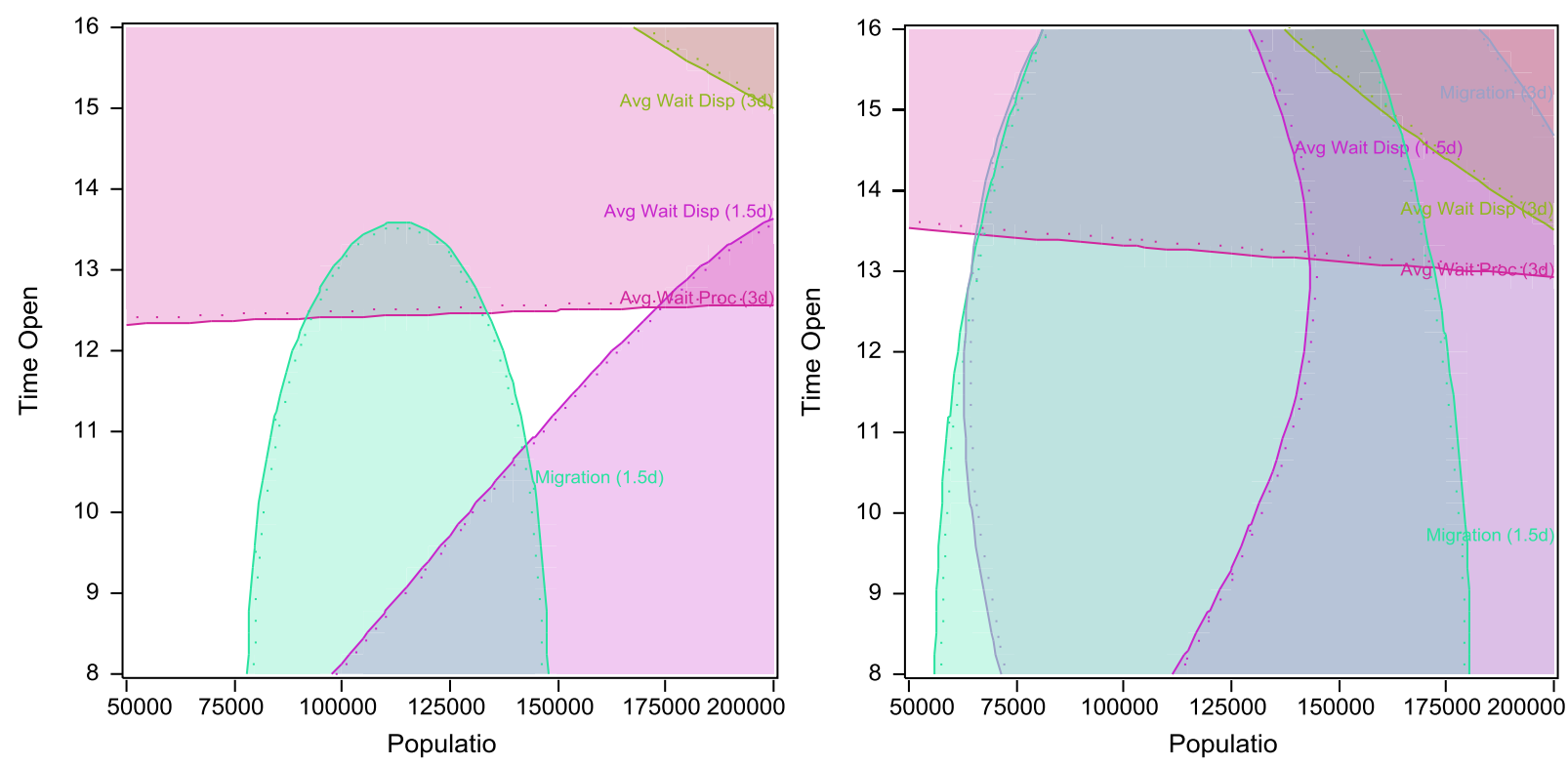

Figure 3: Contour Profiler, Evaluating the Mission Space, 14 centers/3 lines (left), 4 centers/5 lines (right)

An interesting behavior highlighted in the left chart of Figure 3 is the effect that population has on migration and how that affects the time the centers are open. If we disregard the average and maximum wait times for the processing centers, we see that the centers should be open for longer for populations that lie in the middle of the range. This was discussed previously in the prediction profiler results, but is once again highlighted by the contour profiler. As the population grows, if one wants to satisfy all these constraints, there might be areas where it is simply not feasible. In the example highlighted in this figure, the location of the constraints are based on the settings of the other 8 input factors, so if those were to be changed, the constraints would shift. If one was to simply change the number of distribution lines from 14 distribution centers each with 3 distribution lines to 4 distribution centers with 5 lines, the constraint diagram would change to what is depicted on the right of Figure 3. Notice that the shape of the constraints changes and therefore the available space changes. What this results in is that if only 4 centers can be supported, you will only be able to achieve your mission goals when supporting a smaller population.

The value of a tool like the contour profiler, is that the mission planner can quickly dial in the characteristics of the scenario and evaluate different courses of action for the HA/DR mission. The information required to produce this decision support environment is clearly dependent on the models and data used, but if the models and data are available, a decision support environment for HA/DR can be developed for a variety of scenarios, conditions and courses of action.

\section{CONCLUSIONS}

The paper described an agent-based model for humanitarian assistance and disaster relief missions that attempted to evaluate the impact that the number and capacity of distribution and processing centers have on satisfying the need of the population and the unintended consequences, namely crime and migration. The model was analyzed using linear regressions based on the results of designs of experiment. This allowed the authors to efficiently explore the model and obtain the minimum amount of information to create the regressions.

The results indicate that the behaviors produced by the model were fairly linear and agreed with the user's intuition. The regressions created were evaluated using visual decision support tools included in a commercial-off-the-shelf statistical analysis and visualization package. The results obtained indicate that 
given a validated HA/DR model, mission planners could use the approach described to intelligently evaluate a wide range of courses of action and select the most suitable one.

Future work will focus on integrating the model with a logistics model to evaluate the interactions between the supply of the centers and their distribution and characteristics. Recent interest by NATO and the US Department of Defense in conducting HA/DR missions inspired the authors to develop a support framework for course of action evaluation.

\section{ACKNOWLEDGMENTS}

The authors would like to thank Dr. Gary Horne for his contributions to the development of the agent based model. We would also like to thank Dr. Tom Donnelly for his assistance in the development of a Design of Experiments for our analysis. Finally, the authors would like to extend their appreciation to the Office of Naval Research and in particular Ms. Kelly Cooper for sponsoring this research.

\section{REFERENCES}

Buonanno, P. and D. Montolio. 2008. "Identifying the Socio-economic and Demographic Determinants of Crime across Spanish Provinces." International Review of Law and Economics 28.2: 89-97.

Daily Telegraph. 2010a. "Haiti Earthquake: Looting and Gun-fights Break out - Telegraph." Access Date, 29 Nov. 2010. http://www.telegraph.co.uk/news/worldnews/centralamericaandthecaribbean/haiti /7005554/Haiti-earthquake-looting-and-gun-fights-break-out.html.

Daily Telegraph . 2010b. "Haiti Earthquake: Looting and Violence Break out - Telegraph." Access Date, 29 Nov. 2010. http://www.telegraph.co.uk/news/newsvideo/6996287/Haiti-earthquake-looting-andviolence-break-out.html.

Diamond, J. 2005. “Collapse: Why Societies Choose to Fail or Succeed.” New York, NY: Viking Press.

Jones, B. and C. J. Nachtsheim. 2010. "A Class of Screening Designs Robust to Active Second-Order Effects." mODa 9 - Advances in Model-Oriented Design and Analysis 105-112. Physica-Verlag HD.

Malleson, N., A. Evans, and T. Jenkins. 2009. "An Agent-based Model of Burglary." Environment and Planning B: Planning and Design 36: 1103-123.

Pablo, F., D. Lederman, and N. Loayza. 2002. "What Causes Violent Crime?" European Economic Review 46: 1323-357.

Qian, P. Z. G. 2009. "Nested Latin Hypercube Designs,” Biometrika 96: 957-970.

Schrag, J. and S. Scotchmer. 1997. "The Self-reinforcing Nature of Crime." International Review of Law and Economics 17.3: 325-335.

Scorzafave, L. G. and M. Soares. 2009. "Income Inequality and Pecuniary Crimes." Economics Letters 104.1: 40-42.

State of Missouri - State Emergency Management Agency. "LEOP Planning - Appendix 4 to Annex O." Access Date, 04 June 2010. http://sema.dps.mo.gov/Planning /LEOPPlanning.htm.

Wilensky, U. 1999. "NetLogo," Center for Connected Learning and Computer-Based Modeling, Northwestern University. Evanston, IL.http://ccl.northwestern.edu/netlogo/. 


\section{AUTHOR BIOGRAPHIES}

ANDREW TURNER is a Graduate Researcher in the Aerospace Systems Design Laboratory at the Georgia Institute of Technology. He received his B.S. and M.S. in Aerospace Engineering from the Georgia Institute of Technology. He is currently a Doctoral Candidate in Aerospace Engineering at Georgia Tech. His primary research focus is in the development and analysis of complex system-of-system models. His email address is Andrew.Turner@ASDL.gatech.edu.

SANTIAGO BALESTRINI-ROBINSON received his Ph.D. in Aerospace Engineering from the Georgia Institute of Technology. His dissertation work focused on understanding large-scale complex integrated systems through the use of intelligent modeling and simulation. He currently works in the Aerospace Systems Design Laboratory at the Georgia Institute of Technology as a research engineer. His current areas of research are modeling and simulation of complex systems, analysis and design of naval systems, support of decision making under uncertainty, and applied policy analysis and research. His email address is santiago.balestrini@asdl.gatech.edu.

DIMITRI MAVRIS is the Boeing Professor of Advanced Aerospace Systems Analysis at the Guggenheim School of Aerospace Engineering, Georgia Institute of Technology, and the director of its Aerospace Systems Design Laboratory (ASDL). He received his B.S., M.S. and Ph.D. in Aerospace Engineering from the Georgia Institute of Technology. His primary areas of research interest include: advanced design methods, aircraft conceptual and preliminary design, air-breathing propulsion design, multidisciplinary analysis, design and optimization, system of systems, and non-deterministic design theory. Dr. Mavris has also co-authored with his students in excess of 450 publications. Dr. Mavris has received numerous awards and fellowships during his tenure at Georgia Tech. He is currently Fellow of the American Institute of Aeronautics and Astronautics (AIAA) and a Fellow of the National Institute of Aerospace. He was selected as the 1999 Santa Fe Institute Summer fellow. Dr. Mavris is also the recipient of the NSF CAREER award. He has served in several Technical and Program Committees for AIAA and is the US representative to the International Council of the Aeronautical Sciences (ICAS) Board. Academically, Dr. Mavris has been awarded Georgia Tech's prestigious Outstanding Development of Graduate Assistants Award in 1999 and 2004, and in 2000 he received the SAE's Ralph T. Teetor Educator of the Year Award. He is the director of the Center of Excellence in Robust Systems Design and Optimization under the General Electric University Strategic Alliance (GE USA). And he is currently the lead investigator under the Federal Aviation Administration's Center of Excellence under the Partnership for Air Transportation Noise and Emissions Reduction (PARTNER). His email address is dimitri.mavris@aerospace.gatech.edu. 